\title{
Why protective measurement implies the reality of the wave function: Further consolidation
}

\author{
Shan $\mathrm{Gao}^{1,2,3}$ \\ ${ }^{1}$ Institute for the History of Natural Sciences, \\ Chinese Academy of Sciences, Beijing 100190, China \\ ${ }^{2}$ Department of Philosophy, University of Chinese Academy of Sciences \\ ${ }^{3}$ Centre for Philosophy of Natural and Social Science, \\ London School of Economics and Political Science \\ E-mail: gaoshan@ihns.ac.cn.
}

October 12,2016

\begin{abstract}
The existing $\psi$-ontology theorems are based on a simplified assumption of the ontological models framework, according to which when a measurement is performed the behaviour of the measuring device is determined by the ontic state of the measured system immediately before the measurement. In this paper, I give an argument for the reality of the wave function in terms of protective measurements under a more reasonable assumption, according to which the behaviour of the measuring device during a measurement is determined by the total evolution of the ontic state of the measured system during the measurement. In addition, I present a new analysis of how a protective measurement obtains the expectation value of the measured observable in the measured wave function. The analysis strengthens my argument by further clarifying the role the protection procedure plays in a protective measurement.
\end{abstract}

\section{Introduction}

In a previous paper (Gao, 2015), I gave a proof of the reality of the wave function in terms of protective measurements (Aharonov and Vaidman, 1993; Aharonov, Anandan and Vaidman, 1993). The proof does not rely on auxiliary assumptions, and it also applies to deterministic theories such as Bohm's theory. This improves the Pusey-Barrett-Rudolph theorem (Pusey, Barrett and Rudolph, 2012) and other $\psi$-ontology theorems (Colbeck and Renner, 2012; Hardy, 2013; Leifer, 2014). This new proof, like these $\psi$-ontology 
theorems, is also based on the second assumption of the ontological models framework (Harrigan and Spekkens, 2010; Pusey, Barrett and Rudolph, 2012), according to which when a measurement is performed, the behaviour of the measuring device is determined by the ontic state of the measured system (along with the physical properties of the measuring device) immediately before the measurement, whether the ontic state of the measured system changes or not during the measurement. As noted by Gao (2015), however, this is a simplified assumption which may be not valid in general. A more reasonable assumption is that the ontic state of the measured system may be disturbed and thus evolve in a certain way during a measurement, and the behaviour of the measuring device is determined by the total evolution of the ontic state of the system during the measurement, not simply by the initial ontic state of the system. In this paper, I will give an argument for the reality of the wave function in terms of protective measurements under this new assumption. Moreover, I will also clarify the role the protection procedure plays in a protective measurement, which may help understand this result.

\section{The argument}

As pointed out by Gao (2015), the proofs of existing $\psi$-ontology theorems such as the Pusey-Barrett-Rudolph theorem will be invalid under the new assumption. The reason is that under this assumption, even if two nonorthogonal states correspond to the same ontic state initially, they may correspond to different evolution of the ontic state, which may lead to different probabilities of measurement results. Then the proofs of the $\psi$-ontology theorems by reduction to absurdity cannot go through. According to Gao (2015), his direct argument for $\psi$-ontology in terms of protective measurements can go through under the new assumption. He said:

First, according to this assumption, the evolution of the ontic state of a physical system during a protective measurement determines the result of the protective measurement, namely the expectation value of the measured observable in the measured quantum state. Next, since the quantum state of the system keeps unchanged, the evolution of the ontic state of the system is still compatible with the quantum state. This means that even when the system being in the quantum state is not measured, its ontic state may also evolve in this way and such evolution is then a realistic property of the system. Therefore, the expectation value of the measured observable is determined by a realistic property of the measured system, and it is also a realistic property of the system. Then similar to the direct argument given 
in the last section, we can also prove the reality of the quantum state.

However, this argument is seriously flawed. It is true that during a protective measurement the disturbed evolution of the ontic state of the measurd system is compatible with the wave function of the system. But this does not mean that when the system is not measured its ontic state may also evolve in this disturbed way. Thus it still needs to be argued that the disturbed evolution is a realistic property of the system. In the following, I will fix this loophole.

For a protective measurement, there are two sources which may interfere with the spontaneous evolution of the ontic state of the measured system: one is the protection procedure, and the other is the measuring device. However, no matter how they influence the evolution of the ontic state of the measured system, they cannot generate the definite result of the protective measurement, namely the expectation value of the measured observable in the measured wave function, since they contain no information about the measured wave function 11 The measuring device only contains information about the measured observable, and it does not contain information about the measured wave function. Compared with the measuring device, the protection procedure "knows" less. The protection procedure is either a protective potential or a Zeno measuring device. In each case, the protection procedure contains no information about both the measured observable and the measured wave function 2 Thus, if the information about the measured wave function is not contained in the measured system, then the result of a protective measurement cannot be the expectation value of the measured observable in the measured wave function.

On the other hand, if the result of a protective measurement is also determined by the ontic state of the measuring device or the protection procedure through their influences on the spontaneous evolution of the ontic

\footnotetext{
${ }^{1}$ In other words, the properties of the protection setting and the measuring device are independent of the measured wave function.

${ }^{2}$ Certainly, the measurer who does the protective measurement knows more information than that contained in the measuring device and protection procedure. Besides the measured observable, the measurer also knows the measured wave function is one of infinitely many known states (but she needs not know which one the measured wave function is). In the case of protective potential, the measurer knows that the measured wave function is one of infinitely many nondegenerate discrete energy eigenstate of the Hamiltonian of the measured system. In the case of Zeno protection, the measurer knows that the measured wave function is one of infinitely many nondegenerate eigenstates of an observable. Note that this permits the possibility that the measurer can cheat us by first measuring which one amongst these infinitely many states the measured wave function is (e.g. by measuring the eigenvalue of energy for the case of protective potential) and then calculating the expectation value and outputing it through a device. Then the result will have no implications for the reality of the wave function. But obviously this is not a protective measurement.
} 
state of the measured system, then the result may be different for the same measured observable and the same measured wave function. This contradicts the predictions of quantum mechanics, according to which the result of a protective measurement is always the expectation value of the measured observable in the measured wave function.

Therefore, the definite result of a protective measurement, namely the expectation value of the measured observable in the measured wave function, is determined by the spontaneous evolution of the ontic state of the measured system during the measurement. Since the spontaneous evolution of the ontic state of the measured system is an intrinsic property of the system independent of the protective measurement, the expectation value of the measured observable in the measured wave function is also a property of the system. This then proves the reality of the wave function, which can be constructed from the expectation values of a sufficient number of observables.

\section{How does a protective measurement obtain the expectation value?}

In the following, I will present a new analysis of how a protective measurement obtains the expectation value of the measured observable in the measured wave function. The analysis may help understand the above result by further clarifying the role the protection procedure plays in a protective measurement.

By a projective measurement on a single quantum system, one obtains one of the eigenvalues of the measured observable, and the expectation value of the observable can only be obtained as the statistical average of eigenvalues for an ensemble of identically prepared systems. Thus it seems surprising that a protective measurement can obtain the expectation value of the measured observable directly from a single quantum system. In fact, however, this result is not as surprising as it seems to be. The key point is to notice that according to the linear Schrödinger evolution the pointer shift rate at any time during a projective measurement is proportional to the expectation value of the measured observable in the measured wave function at the time. Concretely speaking, for a projective measurement of an observable $A$, whose interaction Hamiltonian is given by the usual form $H_{I}=g(t) P A$, where $g(t)$ is the time-dependent coupling strength of the interaction, and $P$ is the conjugate momentum of the pointer variable, the pointer shift rate at each instant $t$ during the measurement is:

$$
\frac{d\langle X\rangle}{d t}=g(t)\langle A\rangle,
$$

where $X$ is the pointer variable, $\langle X\rangle$ is the center of the pointer wavepacket 
at instant $t$, and $\langle A\rangle$ is the expectation value of the measured observable $A$ in the measured wave function at instant $t$. This pointer shift rate formula indicates that at any time during a projective measurement, the pointer shift after an infinitesimal time interval is proportional to the expectation value of the measured observable in the measured wave function at the time. This result may be more surprising for some people. As is well known, however, since the projective measurement changes the wave function of the measured system greatly, and especially it also results in the pointer wavepacket spreading greatly, the point shift after the measurement does not represent the actual measurement result, and it cannot be measured either. Moreover, even if the point shift after the measurement represents the actual measurement result (e.g. for collapse theories), the result is not definite but random, and it is not the expectation value of the measured observable in the initial measured wave function either.

Then, how to make the expectation value of the measured observable in the measured wave function, which is hidden in the process of a projective measurement, visible in the final measurement result? This requires that the pointer wavepacket should not spread considerably during the measurement so that the final pointer shift is qualified to represent the measurement result, and moreover, the final pointer shift should be also definite. A direct way to satisfy the requirement is to protect the measured wave function from changing as a protective measurement does. Take the Zeno protection scheme as an example. We make frequent projective measurements of an observable $O$, of which the measured state $|\psi\rangle$ is an nondegenerate eigenstate, in a very short measurement interval $[0, \tau]$. For instance, $O$ is measured in $[0, \tau]$ at times $t_{n}=(n / N) \tau, n=1,2, \ldots, N$, where $N$ is an arbitrarily large number. At the same time, we make the same projective measurement of an observable $A$ in the interval $[0, \tau]$ as above. Different from the usual derivation (Aharonov, Anandan and Vaidman, 1993; Gao, 2014; Combes et al, 2015) ${ }^{3}$ here I will calculate the post-measurement state in accordance of the order of time evolution. This will let us see the process of protective measurement more clearly.

The state of the combined system immediately before $t_{1}=\tau / N$ is given by

\footnotetext{
${ }^{3}$ Note that in the usual derivation, the measurement result of a protective measurement, namely the expectation value of the measured observable in the measured wave function, is already contained in the measurement operator which describes the measurement procedure. But this does not imply what the measurement measures is not the property of the measured system, but the property of the measurement procedure such as the protection procedure (cf. Combes et al, 2015). Otherwise, for example, diseases will exist not in patients, but in doctors or expert systems for disease diagnosis.
} 


$$
\begin{aligned}
e^{-\frac{i}{\hbar} \frac{\tau}{N} g\left(t_{1}\right) P A}|\psi\rangle\left|\phi\left(x_{0}\right)\right\rangle= & \sum_{i} c_{i}\left|a_{i}\right\rangle\left|\phi\left(x_{0}+\frac{\tau}{N} g\left(t_{1}\right) a_{i}\right)\right\rangle \\
= & |\psi\rangle\left|\phi\left(x_{0}+\frac{\tau}{N} g\left(t_{1}\right)\langle A\rangle\right)\right\rangle \\
& +\frac{\tau}{N} g\left(t_{1}\right)(A-\langle A\rangle)|\psi\rangle\left|\phi^{\prime}\left(x_{0}+\frac{\tau}{N} g\left(t_{1}\right)\langle A\rangle\right)\right\rangle \\
& +O\left(\frac{1}{N^{2}}\right),
\end{aligned}
$$

where $\left|\phi\left(x_{0}\right)\right\rangle$ is the pointer wavepacket centered in initial position $x_{0},\left|a_{i}\right\rangle$ are the eigenstates of $A$, and $c_{i}$ are the expansion coefficients. Note that the second term in the r.h.s of the formula is orthogonal to the measured state $|\psi\rangle$. Then the branch of the state of the combined system after $t_{1}=\tau / N$, in which the projective measurement of $O$ results in the state of the measured system being in $|\psi\rangle$, is given by

$$
|\psi\rangle\left\langle\psi\left|e^{-\frac{i}{\hbar} \frac{\tau}{N} g\left(t_{1}\right) P A}\right| \psi\right\rangle\left|\phi\left(x_{0}\right)\right\rangle=|\psi\rangle\left|\phi\left(x_{0}+\frac{\tau}{N} g\left(t_{1}\right)\langle A\rangle\right)\right\rangle+O\left(\frac{1}{N^{2}}\right) .
$$

Thus after $N$ such measurements and in the limit of $N \rightarrow \infty$, the branch of the state of the combined system, in which each projective measurement of $O$ results in the state of the measured system being in $|\psi\rangle$, is

$$
|t=\tau\rangle=|\psi\rangle\left|\phi\left(x_{0}+\int_{0}^{\tau} g(t) d t\langle A\rangle\right)\right\rangle=|\psi\rangle\left|\phi\left(x_{0}+\langle A\rangle\right)\right\rangle .
$$

Since the modulus squared of the amplitude of this branch approaches one when $N \rightarrow \infty$, this state will be the state of the combined system after the protective measurement.

By this derivation, it can be clearly seen that the role of the protection procedure is not only to protect the measured wave function from the change caused by the projective measurement, but also to prevent the pointer wavepacket from the spreading caused by the projective measurement. As a result, the pointer shift after the measurement can represent a valid measurement result, and moreover, it is also definite, being natually the expectation value of the measured observable in the initial measured wave function.

\section{Further consolidation}

The above analysis of how a protective measurement obtains its result will strengthen my previous argument for $\psi$-ontology in terms of protective measurements. 
Since the width of the pointer wavepacket keeps unchanged during a protective measurement, and the pointer shift rate at any time during the measurement is proportional to the expectation value of the measured observable in the measured wave function at the time 4 which is the same as the initial measured wave function, we can actually obtain the final measurement result at any time during the protective measurement (when the time-dependent coupling strength is known). This indicates that the result of a protective measurement is determined by the initial ontic state of the measured system, not by the evolution of the ontic state of the system during the measurement, whether spontaneous or disturbed. Thus the second, simplified assumption of the ontological models framework is still valid for protective measurements, so does my previous argument for the reality of the wave function based on this assumption (Gao, 2015).

It has been conjectured that the result of a protective measurement is determined not by the ontic state of the measured system but by the protection procedure, which may lead to a certain evolution of the ontic state of the system that may generate the measurement result (Combes et al, 2015). If this is true, then protective measurements will have no implications for the reality of the wave function. However, as I have argued in the beginning of this section, this conjecture cannot be correct. The essential reason is that the protection procedure does not "know" the measured wave function, and thus it cannot generate the measurement result, the expectation value of the measured observable in the measured wave function 5 In addition, the above analysis also clarifies the role of the protection procedure during a protective measurement. The expectation value of the measured observable in the measured wave function is already hidden in the process of the projective measurement, and what the protection procedure does is to make it visible in the final measurement result by keeping the measured wave function unchanged.

\footnotetext{
${ }^{4}$ Since the pointer shift is always continuous and smooth during a protective measurement, it is arguable that the evolution of the ontic state of the measured system (which determines the pointer shift) is also continuous. Then for an ideal situation where the protective measurement is instantaneous, the ontic state of the measured system will be unchanged after the measurement and my previous argument for $\psi$-ontology in terms of protective measurements will be still valid (Gao, 2015). Note that the evolution of the position of the pointer as its ontic state may be discontinuous in an $\psi$-epistemic model. However, the range of the position variation is limited by the width of the pointer wavepacket, which can be arbitrarily small in principle. Thus such discontinuous evolution cannot be caused by the evolution of the ontic state of the measured system, whether continuous or discontinuous.

${ }^{5}$ Note that in the $\psi$-epistemic models given by Combes et al (2015), it is implicitly assumed that the protection procedure knows the measured wave function. Thus it is not surprising that the models can reproduce the predictions of quantum mechanics for protetcive measurements.
} 


\section{Conclusion}

In this paper, I strengthen my previous argument for the reality of the wave function in terms of protective measurements. The previous argument, like other $\psi$-ontology theorems, is based on a simplified assumption of the ontological models framework, according to which when a measurement is performed the behaviour of the measuring device is determined by the ontic state of the measured system immediately before the measurement, whether the ontic state of the measured system changes or not during the measurement. This simplified assumption may be not valid in general. A more reasonable assumption is that the ontic state of the measured system may be disturbed and thus evolve in a certain way during a measurement, and the behaviour of the measuring device is determined by the total evolution of the ontic state of the system during the measurement. Although the proofs of the existing $\psi$-ontology theorems by reduction to absurdity cannot go through under the new assumption, I argue that my previous proof of $\psi$-ontology in terms of protective measurements can still go through under the assumption. In addition, I present a new analysis of how a protective measurement obtains the expectation value of the measured observable in the measured wave function, and clarify the role the protection procedure plays in a protective measurement. The analysis strengthens my argument for the reality of the wave function in terms of protective measurements.

\section{Acknowledgments}

I am grateful to Matthew Leifer and Matthew Pusey for helpful discussions. Most of this paper was written when I visited the Centre for Philosophy of Natural and Social Science (CPNSS) at the London School of Economics and Political Science. I thank the Center for providing research facilities and Roman Frigg and Laura O'Keefe for their help during my visit. This work is supported by the Top Priorities Program of the Institute for the History of Natural Sciences, Chinese Academy of Sciences.

\section{References}

[1] Aharonov, Y., Anandan, J. and Vaidman, L. (1993). Meaning of the wave function. Phys. Rev. A 47, 4616.

[2] Aharonov, Y. and L.Vaidman (1993). Measurement of the Schrödinger wave of a single particle, Physics Letters A 178, 38.

[3] Colbeck, R., and Renner, R. (2012). Is a system's wave function in one-to-one correspondence with its elements of reality? Phys. Rev. Lett., 108, 150402. 
[4] Combes, J., Ferrie, C., Leifer, M. S., and Pusey, M. F. (2015). Why protective measurement does not establish the reality of the quantum state. arXiv:1509.08893 [quant-ph].

[5] Gao, S. (2014). Protective measurement: An introduction. In S. Gao (eds.). Protective Measurements and Quantum Reality: Toward a New Understanding of Quantum Mechanics. Cambridge: Cambridge University Press. pp.1-12.

[6] Gao, S. (2015). An argument for $\psi$-ontology in terms of protective measurements. Studies in History and Philosophy of Modern Physics, 52, 198-202.

[7] Hardy, L. (2013). Are quantum states real? International Journal of Modern Physics B 27, 1345012.

[8] Harrigan, N. and Spekkens, R. (2010). Einstein, incompleteness, and the epistemic view of quantum states. Found. Phys. 40, 125-157.

[9] Leifer, M. S. (2014). Is the quantum state real? An extended review of $\psi$-ontology theorems, Quanta 3,67-155.

[10] Pusey, M., Barrett, J. and Rudolph, T. (2012). On the reality of the quantum state. Nature Phys. 8, 475-478. 